\title{
GA-SVR and Pseudo-position-aided GPS/INS Integration during GPS Outage
}

\author{
Xinglong Tan ${ }^{1}$, Jian Wang ${ }^{1}$, Shuanggen Jin $^{2,3}$ and Xiaolin Meng ${ }^{4}$ \\ ${ }^{1}$ (School of Environment and Spatial Informatics, China University of Mining and \\ Technology, Xuzhou, China) \\ ${ }^{2}$ (Shanghai Astronomical Observatory, Chinese Academy of Sciences, Shanghai, China) \\ ${ }^{3}$ (Department of Geomatics Engineering, Bulent Ecevit University, Zonguldak, Turkey) \\ ${ }^{4}$ (Institute of Engineering Surveying and Space Geodesy (IESSG), The University of \\ Nottingham, UK) \\ (E-mail: tanxinglong3@126.com)
}

\begin{abstract}
The performance of Global Positioning System and Inertial Navigation System (GPS/INS) integrated navigation is reduced when GPS is blocked. This paper proposes an algorithm to overcome the condition where GPS is unavailable. Together with a parameter-optimised Genetic Algorithm (GA), a Support Vector Regression (SVR) algorithm is used to construct the mapping function between the specific force, angular rate increments of INS measurements and the increments of the GPS position. During GPS outages, the real-time pseudoGPS position is predicted with the mapping function, and the corresponding covariance matrix is estimated by an improved adaptive filtering algorithm. A GPS/INS integration scheme is demonstrated where the vehicle travels along a straight line and around a curve, with respect to both low-speed-stable and high-speed-unstable navigation platforms. The results show that the proposed algorithm provides a better performance when GPS is unavailable.
\end{abstract}
KEY WORDS
1. GPS/INS integrated navigation
2. Pseudo-GPS position.
4. Improved adaptive filtering
3. Support Vector Regression

Submitted: 8 March 2014. Accepted: 10 January 2015. First published online: 13 February 2015.

1. INTRODUCTION. The Global Positioning System (GPS) and Inertial Navigation Systems (INS) have been widely used for navigation. Since GPS satellite signals are subjected to external environmental disturbance, signal blockage is frequently experienced in urban environments, while INS errors accumulate quickly with time. To provide continuous and reliable navigation solutions, the two systems are usually employed together for their complementary characteristics. The degradation of GPS/INS navigation performance is unavoidable when GPS signals are degraded in some areas, such as forests and canyons. Special approaches should be 
developed to overcome this problem, such as GPS integration with additional sensors, e.g., odometers, magnetometers, broadband communication networks (cellular GSM, etc.) or receiver elevation knowledge obtained from a digital terrain model (Danezis and Gikas, 2013; Farrell, 2008; Van Diggelen, 2009).

Artificial intelligence and machine learning can also be used to overcome this problem by taking INS data as the input and the difference from the GPS position as the output. A multi-sensor system integration approach was introduced to fuse data from an INS and GPS hardware-utilising wavelet multi-resolution analysis and Artificial Neural Networks (ANN) (Chiang et al., 2003; Noureldin et al., 2004). A multi-layer neural network was trained to map the vehicle dynamics corresponding to Kalman Filter (KF) states, which can be used to correct INS measurements when GPS measurements are unavailable (Wang et al., 2006). An artificial intelligencebased segmented forward predictor was proposed to update the position and velocity architecture by utilising radial basis function neural networks for the purpose of providing metre-level positioning solutions during GPS outages (Semeniuk and Noureldin, 2006). An auxiliary fuzzy-based model was presented for predicting the KF positioning error states during GPS signal outages (Abdel-Hamid et al., 2007). GPS/INS navigation solutions were calculated intelligently using the ANN based on updating the INS in a Kalman filter structure. To overcome the limitations of multilayer feed-forward neural networks and KF-based schemes, a constructive neural network was proposed to improve positioning accuracy by 55\% during GPS signal outages (El-Sheimy et al., 2008; Huang and Chiang, 2008). However, with neural networks it is difficult to avoid the problems of a local optimal solution, determining the topological structure and the "curse of dimensionality".

The Support Vector Regression (SVR) algorithm is a new regression technology based on the Vapnik-Chervonenkis (VC) dimension theory of statistical learning and the structural risk minimisation principle (Vapnik, 2000). The input is transformed into a high-dimension characteristic space through a nonlinear transformation, and the optimum linearity regression function is sought. The SVR avoids overfitting by choosing a specific hyperplane among the feature spaces and overcomes the major problems faced by typical neural networks, such as local minima, overfitting or over-training, etc. (Frangos et al., 2010), allowing for a much more handsoff training process that is easily deployable and scalable. Xu et al. (2010) established the regression model between the INS output data and the GPS position difference based on the least squares support vector machine, and the results show that the least squares support vector machine is superior to the neural network algorithm. However, the method of acquiring the optimal parameters for SVR model training was not provided.

In this paper, the pseudo-GPS position-aided GPS/INS navigation is presented during GPS outages. With the optimal parameters from genetic algorithms, the SVR algorithm is used to train a regression model between the specific force and angular rate increments of the INS measurements as input and the increments of GPS position as output. The pseudo-GPS position is calculated with the regression model. An improved adaptive filtering algorithm is then designed to estimate the covariance matrix of the pseudo-GPS position in real-time. Finally, the pseudoposition-aided navigation solution is given. The structure of this paper is as follows: Section 2 briefly introduces the theory and model of GPS position-aided INS and 
INS mechanisation equations, Section 3 presents the Genetic Algorithm-Support Vector Regression (GA-SVR) model and its training parameters as well as an optimisation method based on GA. The calculation of the pseudo-position and covariance estimation scheme based on improved adaptive filtering are given in Section 4. Section 5 shows testing results of the pseudo-position-aided low-cost INS scheme, and the conclusions are given in Section 6.

2. CONVENTIONAL GPS/INS INTEGRATED NAVIGATION. A Loosely Coupled (LC) Extended Kalman Filtering (EKF) (Falco et al., 2012; Faruqi and Turner, 2000) is applied for GPS/INS integration, with the states as follows:

$$
\begin{gathered}
\boldsymbol{X}=\left[\delta r_{\mathrm{N}}, \delta r_{\mathrm{E}}, \delta r_{\mathrm{D}}, \delta v_{\mathrm{N}}, \delta v_{\mathrm{E}}, \delta v_{\mathrm{D}}, \delta \phi_{\mathrm{Ro}}, \delta \phi_{\mathrm{Pi}}, \delta \phi_{\mathrm{Ha}}, \nabla_{\mathrm{bx}}, \nabla_{\mathrm{by}}, \nabla_{\mathrm{bz}},\right. \\
\left.\nabla_{\mathrm{fx}}, \nabla_{\mathrm{fy}}, \nabla_{\mathrm{fz}}, \varepsilon_{\mathrm{bx}}, \varepsilon_{\mathrm{by}}, \varepsilon_{\mathrm{bz}}, \varepsilon_{\mathrm{fx}}, \varepsilon_{\mathrm{fy}}, \varepsilon_{\mathrm{fz}}, \delta l_{\mathrm{N}}, \delta l_{\mathrm{E}}, \delta l_{\mathrm{D}}, \delta g_{\mathrm{N}}, \delta g_{\mathrm{E}}, \delta g_{\mathrm{D}}\right]^{\mathrm{T}}
\end{gathered}
$$

where $\delta r_{\mathrm{N}}, \delta r_{\mathrm{E}}, \delta r_{\mathrm{D}}$ are the position errors, $\delta v_{\mathrm{N}}, \delta v_{\mathrm{E}}, \delta v_{\mathrm{D}}$ are the velocity errors, $\delta \phi_{\mathrm{Ro}}$, $\delta \phi_{\mathrm{Pi}}, \delta \phi_{\mathrm{Ha}}$ are the attitude errors, $\nabla_{\mathrm{bx}}, \nabla_{\mathrm{by}}, \nabla_{\mathrm{bz}}$ are the accelerometer biases, $\nabla_{\mathrm{fx}}, \nabla_{\mathrm{fy}}$, $\nabla_{\mathrm{fz}}$ are the accelerometer scale factor errors, $\varepsilon_{\mathrm{bx}}, \varepsilon_{\mathrm{by}}, \varepsilon_{\mathrm{bz}}$ are the gyro drifts, $\varepsilon_{\mathrm{fx}}, \varepsilon_{\mathrm{fy}}$, $\varepsilon_{\mathrm{fz}}$ are the gyro scale factor errors $\delta l_{N}, \delta l_{E}, \delta l_{D}$ represent the GPS antenna to INS lever arm measurement errors and $\delta g_{N}, \delta g_{E}, \delta g_{D}$ denote the gravity uncertainty errors.

For the GPS/INS integrated navigation system, the discrete-time form of the dynamic model is given as:

$$
X_{k}=\Phi_{k, k-1} X_{k-1}+w_{k}
$$

where $x_{k}$ and $x_{k-1}$ are the state vector at epoch $k$ and $k-1$, respectively, $\Phi_{k, k-1}$ is the state transition matrix from epoch $k-1$ to $k$, and $w_{k}$ is the dynamic process noise. The difference in position between GPS measurements and INS measurements in the North-East-Down (NED) frame is regarded as measurements, and the observation equation can be written as:

$$
Z_{k}=H_{k} X_{k}+r_{k}
$$

where $z_{k}$ denotes the difference between INS position and GPS position at epoch $r_{k}, H_{k}=\left[\mathrm{I}_{3 \times 3}, 0_{3} \times 24\right]$ is the observation matrix, and $r_{k}$ represents the measurement noise.

We assume $w_{k}$ and $r_{k}$ satisfy the following conditions:

$$
\left\{\begin{array}{l}
E\left(w_{k}\right)=0, E\left(w_{k} w_{j}^{T}\right)=Q_{k} \delta_{k, j} \\
E\left(r_{k}\right)=0, E\left(r_{k} r_{j}^{T}\right)=R_{k} \delta_{k, j} \\
E\left(w_{k} r_{j}^{T}\right)=0
\end{array}\right.
$$

where $\delta_{k, j}$ is the Kronecker $\delta$-function, $Q_{k}$ is the variance-covariance matrices of states, and $R_{k}$ is the variance-covariance matrices of measurements. The discrete-time Kalman filter is then given by the following equations.

Prediction stage:

$$
\begin{gathered}
\hat{X}_{k, k-1}=\Phi_{k, k-1} \hat{X}_{k-1} \\
P_{k, k-1}=\Phi_{k, k-1} P_{k-1} \Phi_{k, k-1}^{T}+Q_{k-1}
\end{gathered}
$$




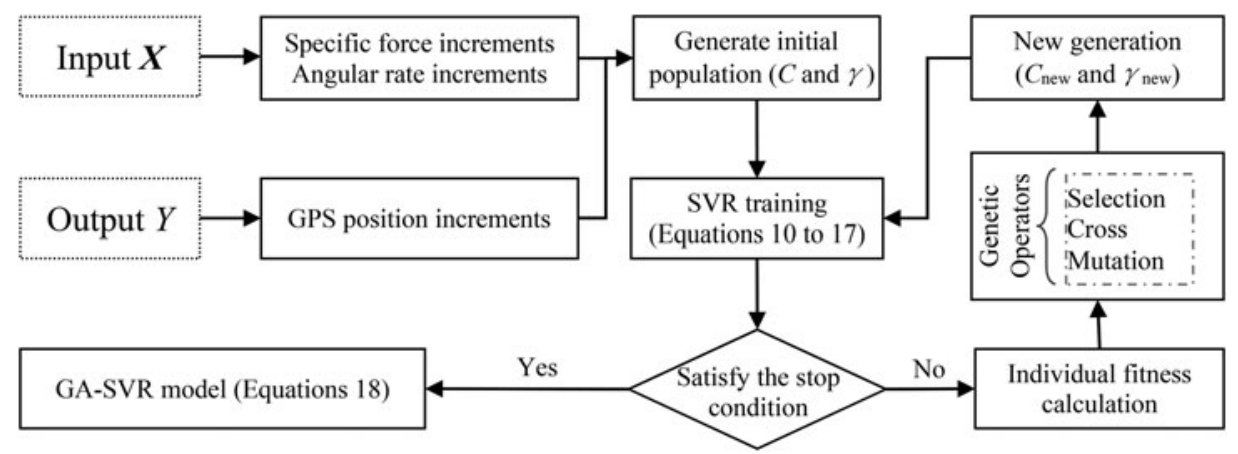

Figure 1. GA-SVR Model training.

Update stage:

$$
\begin{gathered}
K_{k}=P_{k, k-1} H_{k}^{T}\left[H_{k} P_{k, k-1} H_{k}^{T}+R_{k}\right]^{-1} \\
\hat{X}_{k}=\hat{X}_{k, k-1}+K_{k}\left[Z_{k}-H_{k} \hat{X}_{k, k-1}\right] \\
P_{k}=\left[I-K_{k} H_{k}\right] P_{k, k-1}
\end{gathered}
$$

where $\hat{X}_{k, k-1}$ is the a priori state estimate, $P_{k, k-1}$ is the a priori estimate variance-covariance, $K_{k}$ is the Kalman matrix, $\hat{X}_{k}$ is the a posteriori state, and $P_{k}$ is the a posteriori estimate variance-covariance (Bar-Shalom et al., 2001; Chiang et al., 2012; Gikas et al., 1995; Parnian and Golnaraghi, 2010).

3. SVR MODEL TRAINING BASED ON GA. When the vehicle moves steadily without significantly drastic changes, there is a relatively high correlation between the increments of the GPS position and the specific force and angular rate increments of the INS measurements (Wang et al., 2006; Xu et al., 2010). The SVR model of correlation is trained based on the GA in this paper, with the specific force and angular rate increments of the INS measurements as the input $\mathrm{X}$ and the corresponding increments of the GPS position as the output Y of the SVR. The GA is used to search the optimal parameters of the SVR before training. A detailed block diagram of the algorithm is shown in Figure 1.

3.1. Principle of Support Vector Regression. The SVR algorithm seeks the relationship between the input and output for a training set of data $\left(x_{i}, y_{i}\right), i=1,2, \ldots$, $l, x_{i} \in R^{n}, y_{i} \in R$, where $x_{i}$ is the $i$ th input and $y_{i}$ is the corresponding output. The SVR model for nonlinear function estimation has the following representation in the feature space:

$$
f(\boldsymbol{x})=\boldsymbol{\omega}^{\mathrm{T}} \varphi(\boldsymbol{x})+b
$$

where the term $\omega$ is the weight vector. The nonlinear function $\varphi(x)$ maps the input $x$ space to a higher dimensional feature space. The term $b$ is the bias term. 
It is assumed that $\varepsilon$ is the maximum residual between output $y$ and the theoretical value of $f(x)$, so

$$
-\varepsilon<\boldsymbol{y}-f(\boldsymbol{x})<\varepsilon
$$

To obtain the optimum value $\varepsilon_{\min }$ among $\varepsilon$

$$
\begin{aligned}
& \min _{\omega, b} \varepsilon \\
& \text { s.t. }-\varepsilon<\boldsymbol{y}-f(\boldsymbol{x})<\varepsilon, i=1,2, \ldots, l
\end{aligned}
$$

with slack variable $\xi^{(*)}=\left(\xi_{1}, \xi_{1}^{*}, \ldots, \xi_{l}, \xi_{l}^{*}\right) \geq 0$ and penalty parameter $C$ added to Equation (12), the calculation of $\omega$ and $b$ can be altered to the optimisation problem as Equation (13) (Gunn, 1998):

$$
\min _{\omega \in R^{n}, b \in R, \xi^{(*)} \in R^{2 l}} \tau\left(\omega, \xi^{(*)}\right)=\frac{1}{2}\|\boldsymbol{\omega}\|^{2}+\frac{C}{l} \sum_{i=1}^{l}\left(\xi_{i}+\xi_{i}^{*}\right)
$$

To solve the optimisation problem above, the Lagrangian function is constructed:

$$
\begin{gathered}
L(\boldsymbol{\omega}, b, \xi, \alpha)=\frac{1}{2}\|\boldsymbol{\omega}\|^{2}+\frac{C}{l} \sum_{i=1}^{l}\left(\xi_{i}+\xi_{i}^{*}\right)-\sum_{i=1}^{l}\left(\eta_{i} \xi_{i}+\eta_{i}^{*} \xi_{i}^{*}\right) \\
-\sum_{i=1}^{l} \alpha_{i}\left(\varepsilon+\boldsymbol{y}_{i}-(\boldsymbol{\omega} \boldsymbol{x})-b\right)-\sum_{i=1}^{l} \alpha_{i}^{*}\left(\varepsilon-\boldsymbol{y}_{i}+(\boldsymbol{\omega} \boldsymbol{x})+b\right)
\end{gathered}
$$

where $\alpha^{(*)}$ are the Lagrange multipliers $\alpha^{(*)}=\left(\alpha_{1}, \alpha_{1}^{*}, \ldots, \alpha_{l}, \alpha_{l}^{*}\right)^{T} \geq 0$. According to the Wolfe duality theory (Wolfe, 1961), the conditions for optimality are given by:

$$
\left\{\begin{array}{l}
\frac{\partial L}{\partial \omega}=\boldsymbol{\omega}-\sum_{i=1}^{l}\left(\alpha_{i}^{*}-\alpha_{i}\right) \boldsymbol{x}_{i}=0 \\
\frac{\partial L}{\partial b}=\sum_{i=1}^{l}\left(\alpha_{i}-\alpha_{i}^{*}\right)=0 \\
\frac{\partial L}{\partial \xi_{i}}=\frac{C}{l}-\alpha_{i}-\eta_{i}=0 \\
\frac{\partial L}{\partial \xi_{i}^{*}}=\frac{C}{l}-\alpha_{i}^{*}-\eta_{i}^{*}=0
\end{array}\right.
$$

Substituting Equation (15) into Equation (14), Equation (13) can then be expressed as Equation (16):

$$
\begin{aligned}
& \min _{\alpha^{(*)} \in R^{2 l}} \frac{1}{2} \sum_{i, j=1}^{l}\left(\alpha_{i}^{*}-\alpha_{i}\right)\left(\alpha_{j}^{*}-\alpha_{j}\right) \varphi^{\mathrm{T}}\left(\boldsymbol{x}_{i}\right) \varphi\left(\boldsymbol{x}_{j}\right)+\varepsilon \sum_{i=1}^{l}\left(\alpha_{i}^{*}+\alpha_{i}\right)-\sum_{i=1}^{l} y_{i}\left(\alpha_{i}^{*}-\alpha_{i}\right) \\
& \text { s.t. } \sum_{i=1}^{l}\left(\alpha_{i}^{*}-\alpha_{i}\right)=0,0 \leq \alpha_{i}^{*}, \alpha_{i} \leq \frac{C}{l}, i=1,2 \ldots, l
\end{aligned}
$$

Equation (16) belongs to the convex quadratic programming problem, and the feasible region is empty, meaning that the optimal solution of $\bar{\alpha}=\left(\bar{\alpha}_{1}, \bar{\alpha}_{1}^{*}, \ldots, \bar{\alpha}_{l}, \bar{\alpha}_{l}^{*}\right)^{\mathrm{T}}$ in 
Equation (16) is solved. $\omega$ and $b$ can be calculated as follows (Berk, 2008; Joachims, 2002; Williams, 2011):

$$
\begin{gathered}
\boldsymbol{\omega}=\sum_{i=1}^{l}\left(\bar{\alpha}_{i}^{*}-\bar{\alpha}_{i}\right) \varphi\left(\boldsymbol{x}_{i}\right) \\
\left\{\begin{array}{l}
b=\boldsymbol{y}_{i}-\boldsymbol{\omega} \varphi\left(\boldsymbol{x}_{i}\right)-\varepsilon, 0<\bar{\alpha}_{i}^{*}<C \\
b=\boldsymbol{y}_{i}-\boldsymbol{\omega} \varphi\left(\boldsymbol{x}_{i}\right)+\varepsilon, 0<\bar{\alpha}_{i}<C
\end{array}\right.
\end{gathered}
$$

As a result, the SVR model for nonlinear function estimation becomes:

$$
f(\boldsymbol{x})=\sum_{i=1}^{l}\left(\bar{\alpha}_{i}^{*}-\bar{\alpha}_{i}\right) K\left(\boldsymbol{x}_{i}, \boldsymbol{x}\right)+b
$$

where $K\left(x_{i}, x\right)=\varphi^{T}\left(x_{i}\right) \varphi\left(x_{j}\right)$ is a positive definite kernel matrix. Note that the Radial Basis Function (RBF) has an advantage in processing linearly inseparable data, and therefore the RBF kernel $\left(K\left(x_{i}, x_{j}\right)=\exp \left(-\gamma\left\|x_{i}-x_{j}\right\|^{2}\right)\right)$ is chosen as the kernel function. The $\gamma$ is the kernel width: small and kernel width may cause over-fitting, and a large kernel width may cause under-fitting (Chang et al., 2005). A small penalty parameter $(C)$ leads to over-fitting and a large one brings about under-fitting (Alpaydin, 2004). The performance of SVR with Gaussian RBF kernel is sensitive to the kernel width $(\gamma)$ and penalty parameter $(C)$. Several methods can be used to obtain the optimal $\gamma$ and $C$, e.g., bootstrapping, VC bounds statistical learning theory, and inference or Bayesian learning methods (Cristianini and Ricci, 2008; Kecman, 2005). Genetic algorithms are developed in this paper, shown in the next section.

3.2. Parameter optimisation based on genetic algorithms. Genetic algorithms are a family of computational models inspired by evolution. These algorithms encode a potential solution to a specific problem on a simple chromosome-like data structure, and they apply recombination operators to these structures in a way that preserves critical information (Goldberg and Holland, 1988). With respect to $r, C$ of SVR, the solutions of the parameter optimisation problem can be expressed as follows.

Step 1: Encoding. Note that there is only one change between two adjacent numbers and the grey code is developed in this paper. The relationship between binary code $B$ and grey code $G$ is:

$$
\left\{\begin{array}{l}
B_{n-1}=G_{n-1} \\
G_{i}=G_{i+1} \oplus G_{i}, i=0,1,2, \ldots, n-2
\end{array}\right.
$$

where $\oplus$ represents the XOR operator.

Step 2: Initialisation. Set the range of parameters $0 \leq r \leq 1000,0<C \leq 500,20$ chromosomes of each parameter $\gamma, C$ are generated randomly and the maximal genetic generation is 200 .

Step 3: Fitness calculation of the individual. The fitness function is the basis of the optimisation to evaluate the quality of the individual. The Root Mean Square (RMS) of the SVR-trained residual is calculated based on K-fold cross-validation with the 20 chromosomes. Descending through the chromosomes according to the RMS, the fitness of each chromosome is then shown in Equation (20):

$$
\text { Fitn } V(P o s)=2-s p+2 \times(s p-1) \times \frac{P o s-1}{N-1}
$$




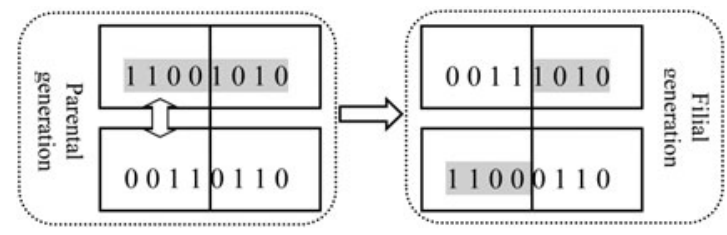

Figure 2. Single-point crossover.

where $s p$ is the assigned press difference, Pos is the position of the chromosomes and $N$ is the population size. Fitn $V \in[1,2]$.

Step 4: Genetic operators.

Selection: The population of the next generation is formed by means of a probabilistic reproduction process. Individuals with a higher fitness usually have a greater chance for the next generation. The selected probability $P_{s i}$ of the $i$ th chromosome $x_{i}$ is shown in Equation (21).

$$
P_{s i}=f_{i} / \sum_{j=1}^{N} f_{j}
$$

where $N$ denotes the size of the population and $f_{i}$ the fitness function of chromosome $x_{i}$.

Crossover: Crossing over tends to enable the evolutionary process to move toward promising regions of the search space. The next generation is formed between two selected individuals, called parents, by exchanging parts of their strings. Single-point crossover is developed with the probability of $0 \cdot 7$ in this paper as seen in Figure 2.

Mutation: Mutation is used to search for additional problem space and to avoid the local convergence of GA. For each bit in the population in this paper, 'mutate' changes the bit value with a low probability of 0.05 .

Step 5: End the GA procedure, and output the optimal chromosome if the genetic generation reaches the maximum value, else, go to step 3.

4. PSEUDO-GPS POSITION-AIDED INS NAVIGATION. When GPS signals are available, the LC strategy, including an EKF, is adopted to combine the GPS and INS values to estimate navigation solutions. The INS errors in 24 states estimated by EKF correct the INS model in real-time. Simultaneously, the regression model is trained based on the GA-SVR, which maps the increments of the GPS position with the specific force and angular rate increments of the INS measurements. If the GPS signals are unavailable, the pseudo-GPS position can be estimated based on the trained model and the specific force and angular rate increments of the INS measurements. An improved adaptive filtering is designed to estimate the covariance matrix of the pseudo-GPS positions in real time. The INS errors estimated by improved adaptive filtering correct the INS model. The pseudo-GPS position-aided navigation solutions are then calculated as shown in Figure 3.

4.1. Pseudo-GPS position calculation. Assume that $t_{i}$ is the epoch before GPS becomes unavailable, $P_{i}$ is the corresponding position of the GPS. $\Delta \mathrm{t}$ is the sampling interval of the GPS measurements. At the next epoch $t_{i+1}\left(t_{i+1}=t_{i}+\Delta t\right)$, the GPS position increments $\Delta P_{j}$ can be derived using the trained GA-SVR model, specific force increments $\int_{t_{i}}^{t_{i+1}} \boldsymbol{f}_{i b}^{b} d t$ and angular rate increments $\int_{t_{i}}^{t_{i+1}} \boldsymbol{\omega}_{i b}^{b} d t$ (according to Equation (18)). 


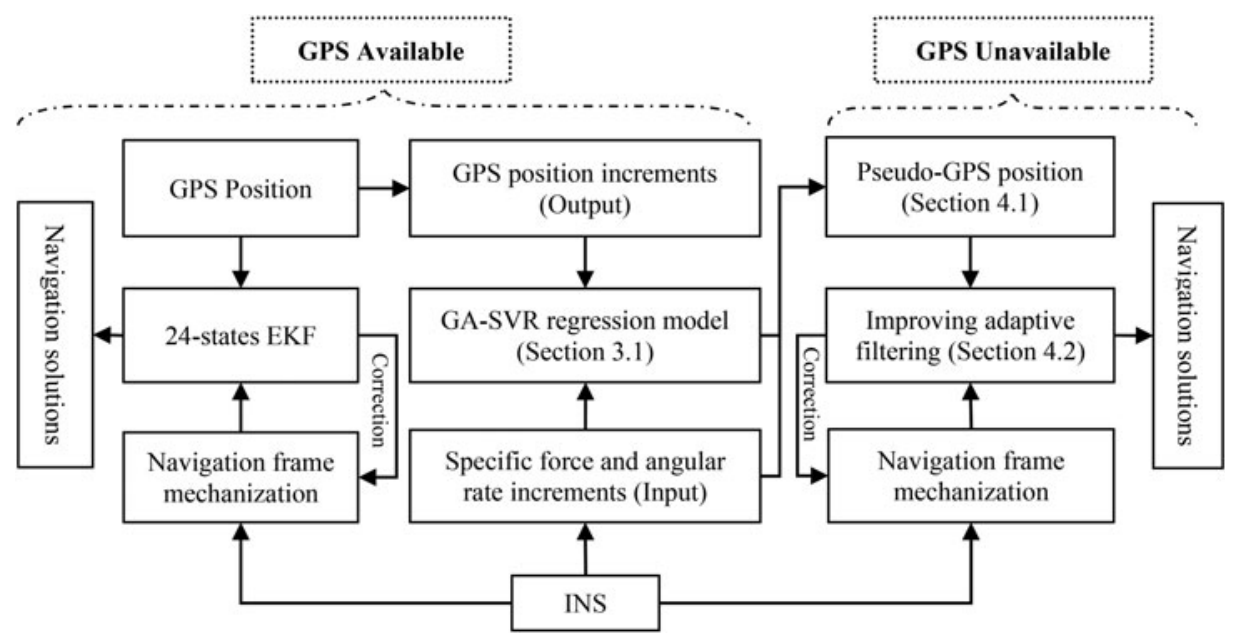

Figure 3. GA-SVR-Based Pseudo-Position-aided INS Navigation algorithm.

The pseudo-GPS position at epoch $t_{i+1}$ can then be obtained as:

$$
\boldsymbol{P}_{j}=\boldsymbol{P}_{i}+\Delta \boldsymbol{P}_{j}
$$

After $n$ intervals, the final pseudo-GPS position at epoch $t_{k}\left(t_{k}=t_{i}+n \Delta t\right)$ is obtained as:

$$
\boldsymbol{P}_{k}=\boldsymbol{P}_{i}+\sum_{t=1}^{n} \Delta \boldsymbol{P}_{t}
$$

4.2. Improved adaptive filtering. The EKF is disabled due to the absence of the covariance matrix of the pseudo-GPS position from the GA-SVR algorithm. In this paper, an improved adaptive filtering algorithm is proposed by combining SageHusa Adaptive Filtering (SHAF) with robust filtering. The SHAF can estimate the covariance matrix in real time according to the innovation to improve the estimation accuracy (Ding et al., 2007). The predicted pseudo-GPS positions inevitably contain big errors/biases, so that a robust algorithm which can detect and solve the errors is needed, such as the equivalent weight method (Yuanxi, 1994) or Receiver Autonomous Integrity Monitoring (RAIM) (Hewitson and Wang, 2007, 2010).

\section{- Sage-Husa adaptive filtering}

The innovation sequence is defined as Equation (24):

$$
\boldsymbol{v}_{k}=\boldsymbol{Z}_{k}-\boldsymbol{H}_{k} \hat{\boldsymbol{X}}_{k, k-1}
$$

The predicted error covariance matrix from the innovation sequence is:

$$
E\left(\boldsymbol{v}_{k} \boldsymbol{v}_{k}^{T}\right)=\boldsymbol{H}_{k} \boldsymbol{P}_{k, k-1} \boldsymbol{H}_{k}+\boldsymbol{R}_{k}
$$

There is clearly a relationship in Equation (25) to estimate $R_{k}$. However, it requires a limited number (called 'estimation window size') of innovation samples to calculate $E\left(v_{k} v_{k}^{T}\right)$. Considering the number of pseudo-measurements, we use both a priori knowledge $R_{k-1}$ and innovation $v_{k} v_{k}^{T}$ to estimate the covariance matrix $R_{k}$ as follows 
(Lu et al., 2007; Sage and Husa, 1969):

$$
\boldsymbol{R}_{k}=\left(1-d_{k}\right) \boldsymbol{R}_{k-1}+d_{k}\left(\boldsymbol{v}_{k} \boldsymbol{v}_{k}^{T}-\boldsymbol{H}_{k} \boldsymbol{P}_{k, k-1} \boldsymbol{H}_{k}^{T}\right)
$$

where $d_{k}=\frac{1-e}{1-e^{k}}, 0<e<1, e$ is the forgetting factor.

$$
d_{k}=\frac{1-e}{1-e^{k}}, 0<e<1
$$

\section{- Robust filtering}

The residual sequence is defined as:

$$
\boldsymbol{\varepsilon}_{k}=\boldsymbol{Z}_{k}-\boldsymbol{H}_{k} \hat{\boldsymbol{X}}_{k}
$$

Then the mean square error factor $\hat{\sigma}_{0}$ is calculated with the median method as:

$$
\hat{\sigma}_{0}=\operatorname{med}_{i}\left\{\left|\sqrt{P_{i}} \varepsilon_{i}\right|\right\} / 0.6745
$$

where $\varepsilon_{i}$ is the $i$ th element of the residual sequence with the weight $P_{i}$. The standardised residual $s_{i}$ of $\varepsilon_{i}$ as:

$$
s_{i}=\left|\sqrt{P_{i}} \varepsilon_{i}\right| / \hat{\sigma}_{0}
$$

Robust factors $\gamma_{i}$ based on IGGIII weight function (Yuanxi, 1994) are constructed as:

$$
\gamma_{i}=\left\{\begin{array}{l}
1, s_{i} \leq k_{0} \\
\frac{k_{0}}{s_{i}} \times\left[\frac{k_{1}-s_{i}}{k_{1}-k_{0}}\right]^{2}, k_{0}<s_{i} \leq k_{1} \\
10^{-30}, s_{i}>k_{1}
\end{array}\right.
$$

where $k_{0}, k_{1}$ are threshold value and $k_{0}=1.0 \sim 1.5, k_{1}=2.5 \sim 8.0$.

If $s_{i} \leq k_{0}$,we think that the $i$ th pseudo-GPS position has no error; if $k_{0}<s_{i} \leq k_{1}$, we think that the $i$ th pseudo-GPS position has small error; and if $s_{i}>k_{1}$, we think that the $i$ th pseudo-GPS position has big error. To reduce the impact of the big errors/biases to the navigation solutions, the covariance matrix of pseudo-GPS positions is amplified with robust factors as follows:

$$
\hat{R}_{i}=\hat{R}_{i} / \gamma_{i}
$$

5. TEST RESULTS. Two sets of Leica 1200 Base and Rover GPS Systems and SPAN-CPT INS units are used. Firstly initial alignment is done with the supporting software of SPAN-CPT, and then the raw IMU data from SPAN-CPT and GPS data from Leica are collected to validate the proposed algorithm in this paper. The sensor specifications of the SPAN-CPT are listed in Table 1.

If GPS signals are available, the loosely coupled strategy is adopted to calculate the navigation solutions of the GPS/INS integrated system based on EKF. Figure 4a shows the position errors of the GPS/INS navigation solution for the latitude, longitude and height when GPS signals are available. The lever arm between GPS antenna phase centre and IMU navigation centre directly affects the output position 
Table 1. INS technical specifications.

\begin{tabular}{lll}
\hline & Gyro & Accelerometer \\
\hline Range & $\pm 375^{\circ} / \mathrm{s}$ & $\pm 10 \mathrm{~g}$ \\
Bias & $20^{\circ} / \mathrm{hr}$ & $50 \mathrm{mg}$ \\
Bias Stability & $\pm 1 \% / \mathrm{hr}$ & $\pm 0 \cdot 75 \mathrm{mg}$ \\
Scale Factor & $1500 \mathrm{ppm}$ & $4000 \mathrm{ppm}$ \\
Random Walk & $0 \cdot 0667^{\circ} / \sqrt{\mathrm{hr}}$ & $60 \mu \mathrm{g} / \sqrt{\mathrm{Hz}}$ \\
\hline
\end{tabular}
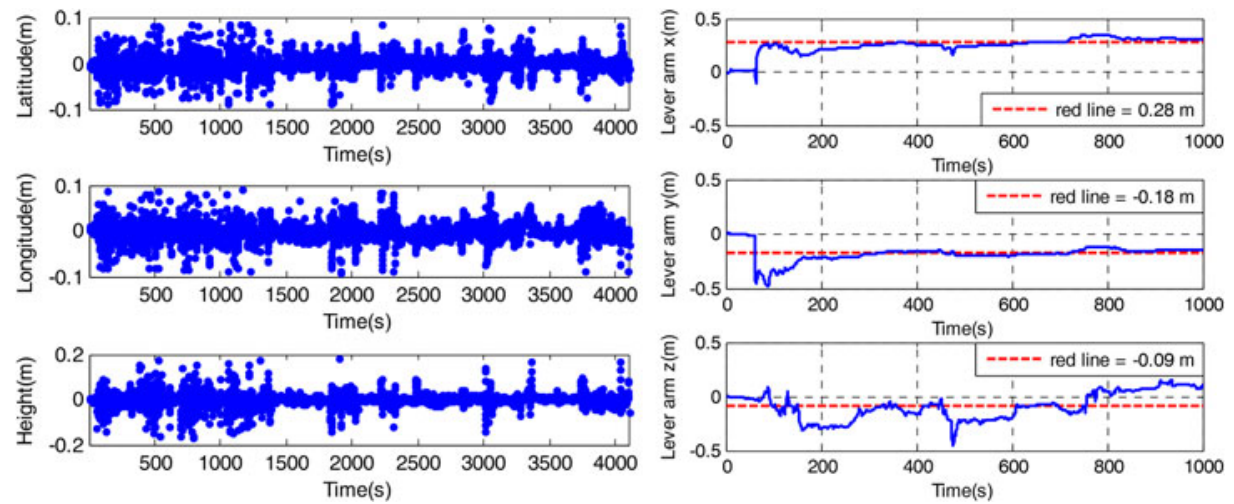

Figure 4. (a) Navigation solution errors (Left). (b) Lever arm estimation (Right).

in a GPS/INS integrated system. According to the algorithm (Geng et al., 2011; Tang et al., 2009), Figure $4 \mathrm{~b}$ shows the estimated lever arm, the red line represents true values.

The feasibility of the algorithm is verified using three tests. The data were recorded for post processing. Test 1 moved along a straight line with respect to a low-speedstable navigation platform, Test 2 moved along a straight line with respect to a high-speed-unstable navigation platform, and Test 3 moved along a curve. Trajectories of these three tests can be seen in Figure 5.

Test 1: 4100 seconds of RTK-GPS $(1 \mathrm{~Hz})$ and IMU $(100 \mathrm{~Hz})$ data were collected when the vehicle moved at a speed of $20 \mathrm{~km} / \mathrm{h}$. Navigation solutions between the 1580 th and 1700th seconds are provided with the proposed algorithm, assuming that the GPS signal was unavailable during that time. With a similar motion state, 101 groups of data from the 1380th to 1480th seconds were chosen as the GA-SVR training samples. The data from 1481st to 1579th seconds were not used because the vehicle was forced to stop at a crossroad.

Test 2: 2100 seconds of RTK-GPS $(1 \mathrm{~Hz})$ and IMU $(100 \mathrm{~Hz})$ data were measured at a speed of $70 \mathrm{~km} / \mathrm{h}$. Navigation solutions between the $561 \mathrm{st}$ and 800 th seconds were solved with the algorithm, assuming that the GPS signal was unavailable during that time. With a similar motion state, 201 groups of data from the 360th to the 560th seconds were chosen as the GA-SVR training samples.

Test 3: A set of data was selected when the vehicle moved along a curve at a speed of $20 \mathrm{~km} / \mathrm{h}$. Navigation solutions between the $943 \mathrm{rd}$ and 1023rd seconds are provided 

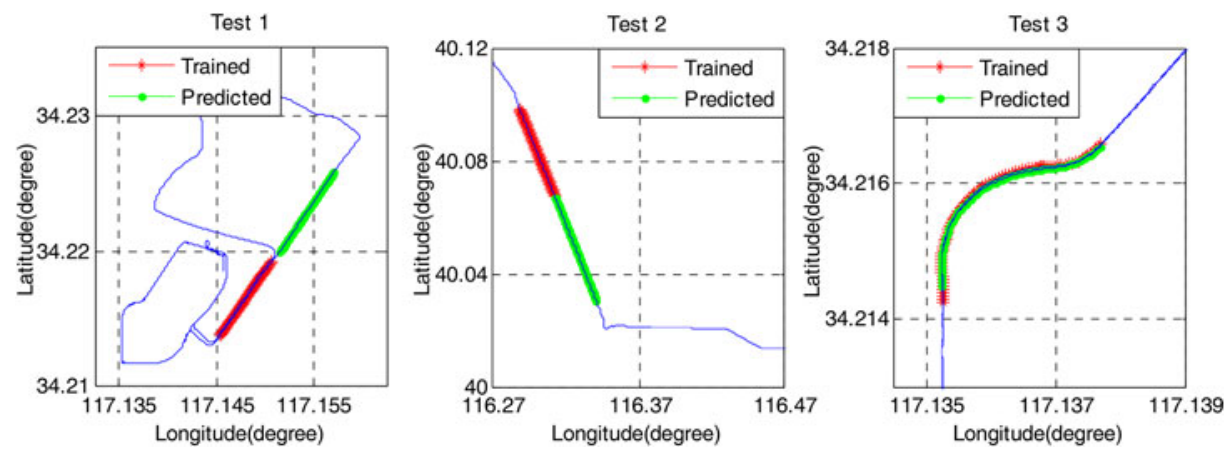

Figure 5. Trajectories for trained and predicted data. (a) Test 1 (Left). (b) Test 2 (Middle). (c) Test 3 (Right).
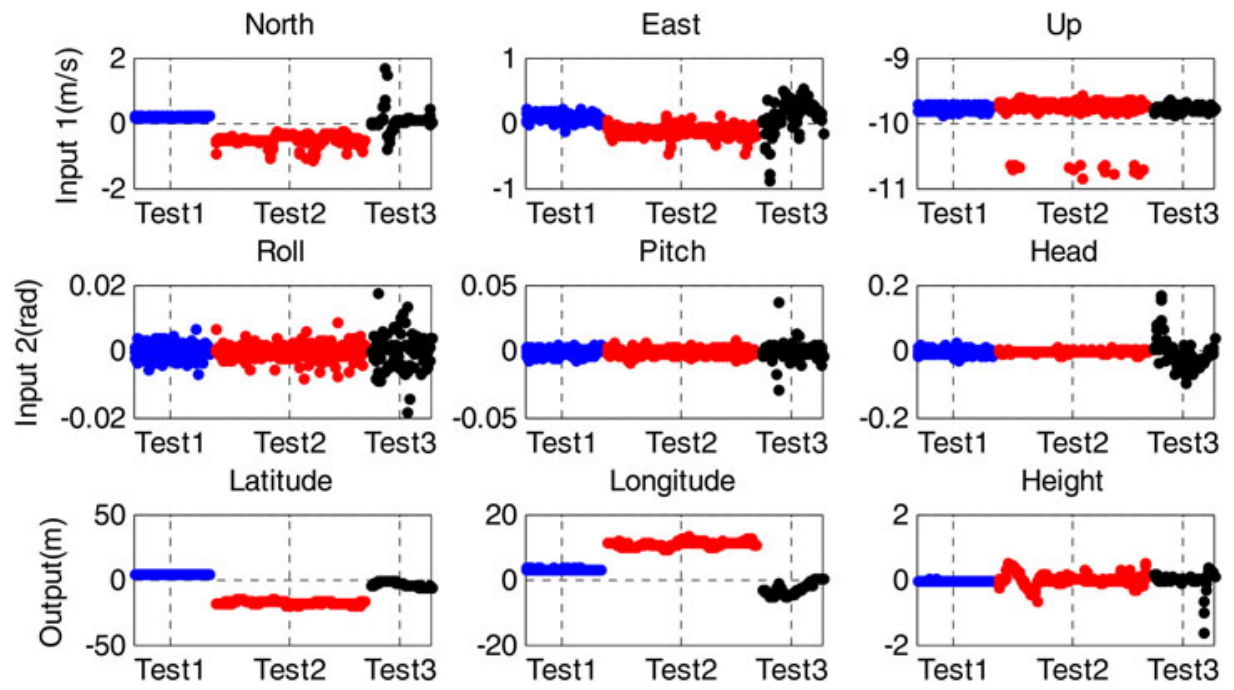

Figure 6. GA-SVR training data of three tests.

with the proposed algorithm, assuming that the GPS signal was unavailable during that time. With a similar motion state, 81 groups of data from the 313 rd to the 393rd seconds were chosen as the GA-SVR training samples.

5.1. GPS position increments based on the GA-SVR. Figure 6 shows the training data of the three tests. Note that the specific force and angular rate increments of the INS measurements are multiplied by the sample interval $0.01 \mathrm{~s}$. In Test 1 and Test 2, the roll and pitch of the angular rate are no more than 0.005 radians in magnitude, and the heading is no more than 0.02 radians, which illustrates that the direction of movement of the test vehicle remains stable. The specific force increments in Test 1 appear smoother than those in Test 2, and the result for the change of the GPS position increments in Test 1 is more stable than that for Test 2. From the increments of heading, latitude and longitude, it is obvious that Test 3 passed through a curve. 

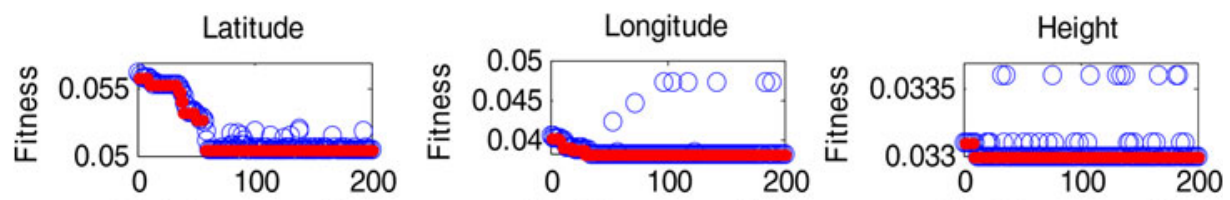

Evolution generations

Latitude

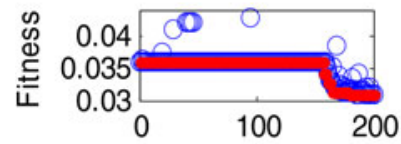

Evolution generations

Latitude
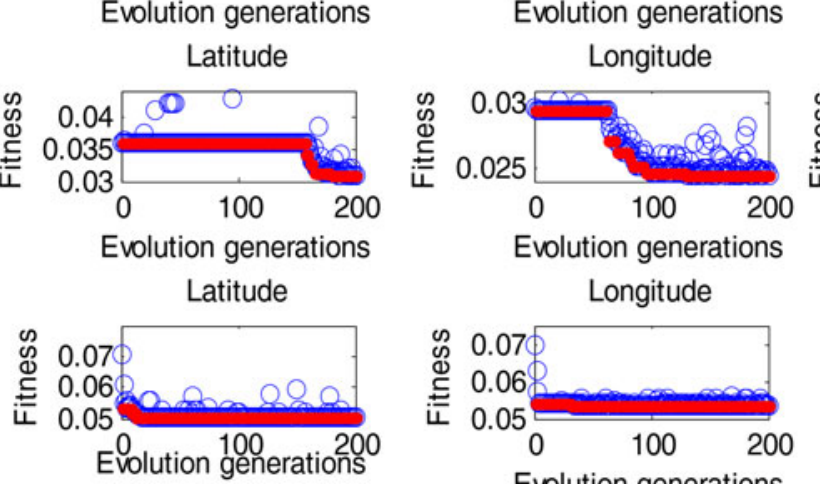

Evolution generations

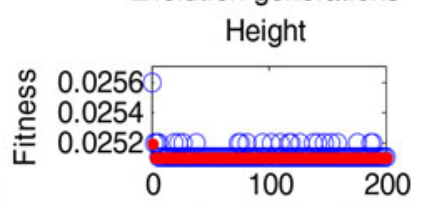

Evolution generations

Evolution generations

Longitude
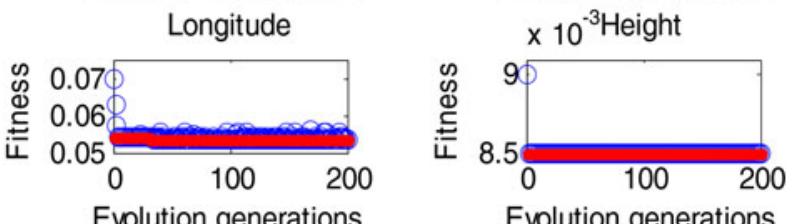

Evolution generations

Evolution generations

Average - Best

Figure 7. Genetic algorithm fitness curves. (a) Test 1 (Top). (b) Test 2 (Middle). (c) Test 3 (Bottom).

Table 2. Results of GA-SVR.

\begin{tabular}{|c|c|c|c|c|c|c|c|}
\hline \multirow[b]{2}{*}{ Test } & \multirow[b]{2}{*}{ Direction } & \multicolumn{2}{|c|}{ Results of GA } & \multirow{2}{*}{$\begin{array}{l}\text { Number } \\
\text { of support } \\
\text { vectors }\end{array}$} & \multirow[b]{2}{*}{ Bias } & \multirow{2}{*}{$\begin{array}{l}\text { RMS error of } \\
\text { trained }(\mathrm{m})\end{array}$} & \multirow{2}{*}{$\begin{array}{l}\text { RMS error of } \\
\text { predicted }(\mathrm{m})\end{array}$} \\
\hline & & $\mathrm{C}$ & $\gamma$ & & & & \\
\hline & latitude & 0.576 & $22 \cdot 020$ & 101 & $-0 \cdot 463$ & $7 \cdot 46 e-3$ & $0 \cdot 083$ \\
\hline \multirow[t]{3}{*}{1} & longitude & $3 \cdot 194$ & $29 \cdot 592$ & 101 & $-0 \cdot 551$ & $3 \cdot 44 \mathrm{e}-4$ & $0 \cdot 111$ \\
\hline & height & $7 \cdot 750$ & $107 \cdot 273$ & 101 & $-0 \cdot 456$ & $5 \cdot 26 e-5$ & $0 \cdot 024$ \\
\hline & latitude & $312 \cdot 228$ & 0.007 & 191 & $-0 \cdot 173$ & 0.994 & $0 \cdot 835$ \\
\hline \multirow[t]{3}{*}{2} & longitude & $354 \cdot 014$ & $0 \cdot 001$ & 192 & $-1 \cdot 371$ & 0.647 & $0 \cdot 515$ \\
\hline & height & $302 \cdot 118$ & $0 \cdot 012$ & 187 & $3 \cdot 501$ & 0.003 & 0.005 \\
\hline & latitude & $22 \cdot 254$ & $116 \cdot 920$ & 77 & $-0 \cdot 511$ & $0 \cdot 163$ & $0 \cdot 295$ \\
\hline \multirow[t]{2}{*}{3} & longitude & $6 \cdot 792$ & $141 \cdot 278$ & 80 & -0.384 & $0 \cdot 113$ & $0 \cdot 201$ \\
\hline & height & $51 \cdot 796$ & $780 \cdot 042$ & 64 & $-0 \cdot 830$ & $0 \cdot 166$ & $0 \cdot 017$ \\
\hline
\end{tabular}

Figure 7 shows the process of seeking the optimal parameters $\gamma$ and C in an SVR based on genetic algorithms. The iteration-stopping criterion is defined as a difference between two adjacent fitness levels of less than 0.001. The iterations in latitude, longitude, and height are: 57, 35, and 9, respectively, in Test 1; 193, 130, and 2, respectively, in Test 2; 13, 32, and 2, respectively, in Test 3. A faster convergence rate is achieved in the height direction for tests, and the slowest convergence rate arises in the latitude in Test 2, where the changes in the GPS position increments are the largest. The optimal parameters $\gamma$ and $\mathrm{C}$ can be seen in Table 2 .

Based on SVR theory, the sample data are trained to construct regression models with the optimal parameters $\gamma$ and C in the SVR determined by genetic algorithms. Figure 8 shows the results and deviations of trained and predicted GPS position 

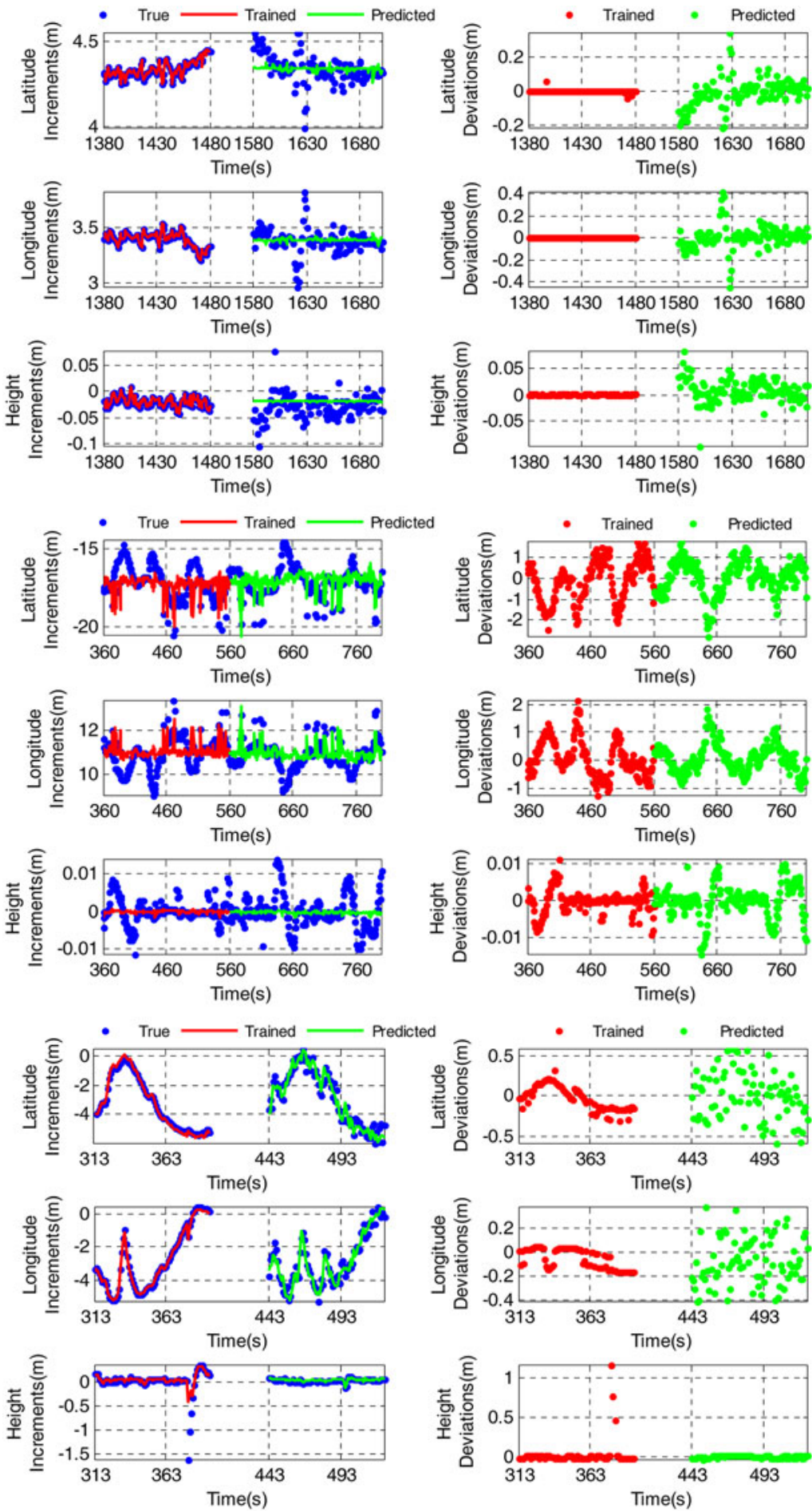

Figure 8. GA-SVR training results of Latitude, Longitude, and Height. (a) Test 1 (Top). (b) Test 2 (Middle). (c) Test 3 (Bottom). 
Test 1
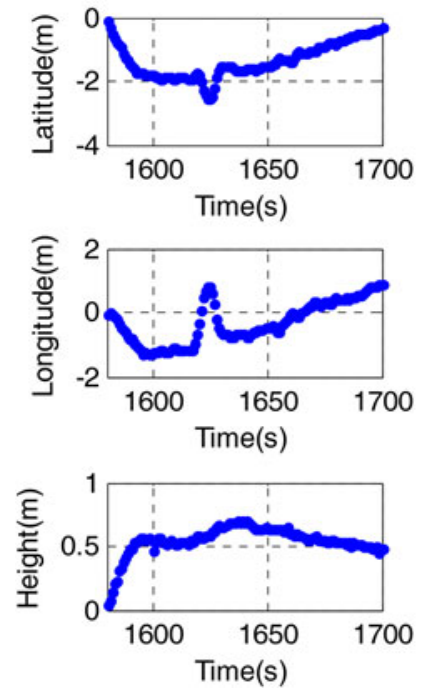

Test 2
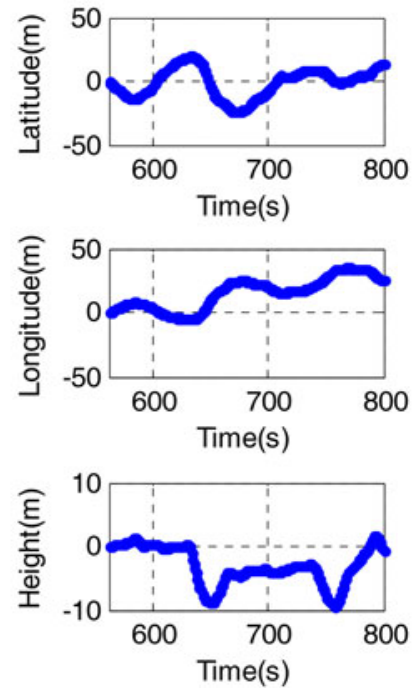

Test 3
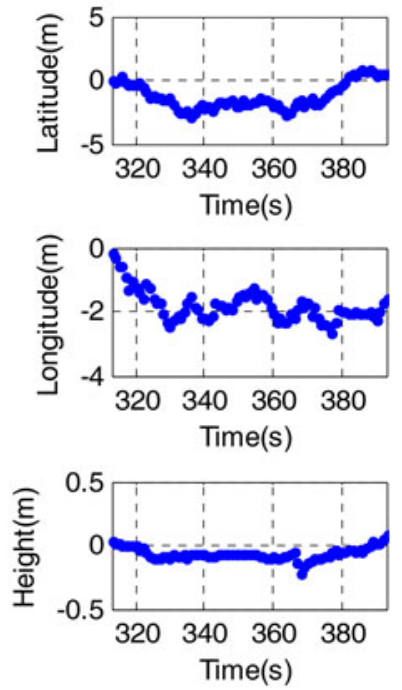

Figure 9. Pseudo-GPS position comparison and deviations. (a) Test 1 (Left). (b) Test 2 (Middle). (c) Test 3 (Right).

increments based on the GA-SVR algorithm. With a more smooth and stable state, the trained and predicted accuracy in Test 1 and Test 3 is much better than that in Test 2. The RMS of the errors of the three tests is shown in Table 2.

5.2. Pseudo-GPS position-aided navigation. The pseudo-GPS position is calculated by adding the predicted GPS position increments to the GPS position (recorded before the GPS outage), as shown in Section 4.1. Figure 9 shows the pseudo-GPS position and its deviations for the three tests. Note that latitude deviations are transformed to metres by multiplying the radius of the curvature in the meridian, and longitude deviations are transformed by multiplying the radius of the curvature in the prime vertical and cosine of the latitude. The RMS of the latitude, longitude, and height deviations are $1.440 \mathrm{~m}, 0.717 \mathrm{~m}$, and $0.561 \mathrm{~m}$, respectively in Test $1,11.641 \mathrm{~m}, 20.148 \mathrm{~m}$, and $4.350 \mathrm{~m}$, respectively in Test 2, and $1.576 \mathrm{~m}, 1.874 \mathrm{~m}$, and $0.077 \mathrm{~m}$, respectively in Test 3 . The accuracy of the pseudo-GPS position in Test 1 and Test 3 is obviously much higher than that in Test 2 due to low speeds and smooth operation.

INS/RTK-GPS stands for the conventional GPS/INS loosely coupled integration algorithm when GPS signals are available; INS/GA-SVR means improved adaptive filtering with the pseudo-GPS positions during the absence of GPS signals; INS-only represents the navigation system depending solely on the equipped INS. Figure 10 shows deviations in the comparisons of the INS/RTK-GPS, INS/GA-SVR, and INS-only algorithms in three dimensions. The results indicate that deviations for INS-only are drifted to $27 \mathrm{~m}$ in 120 seconds when the GPS is unavailable, but the RMS of the deviation with INS/GA-SVR is $1.699 \mathrm{~m}$ with a maximum deviation of no more than $2.734 \mathrm{~m}$ in Test 1 . The performance is very stable. In Test 2, the deviations in the INS-only drift to $119 \mathrm{~m}$ in 240 seconds, while the RMS of the deviation with INS/GA-SVR is $24.026 \mathrm{~m}$, with a maximum deviation of less than $36.403 \mathrm{~m}$. Navigation solutions undulate frequently from 560 680 seconds and are better than 
Test 1
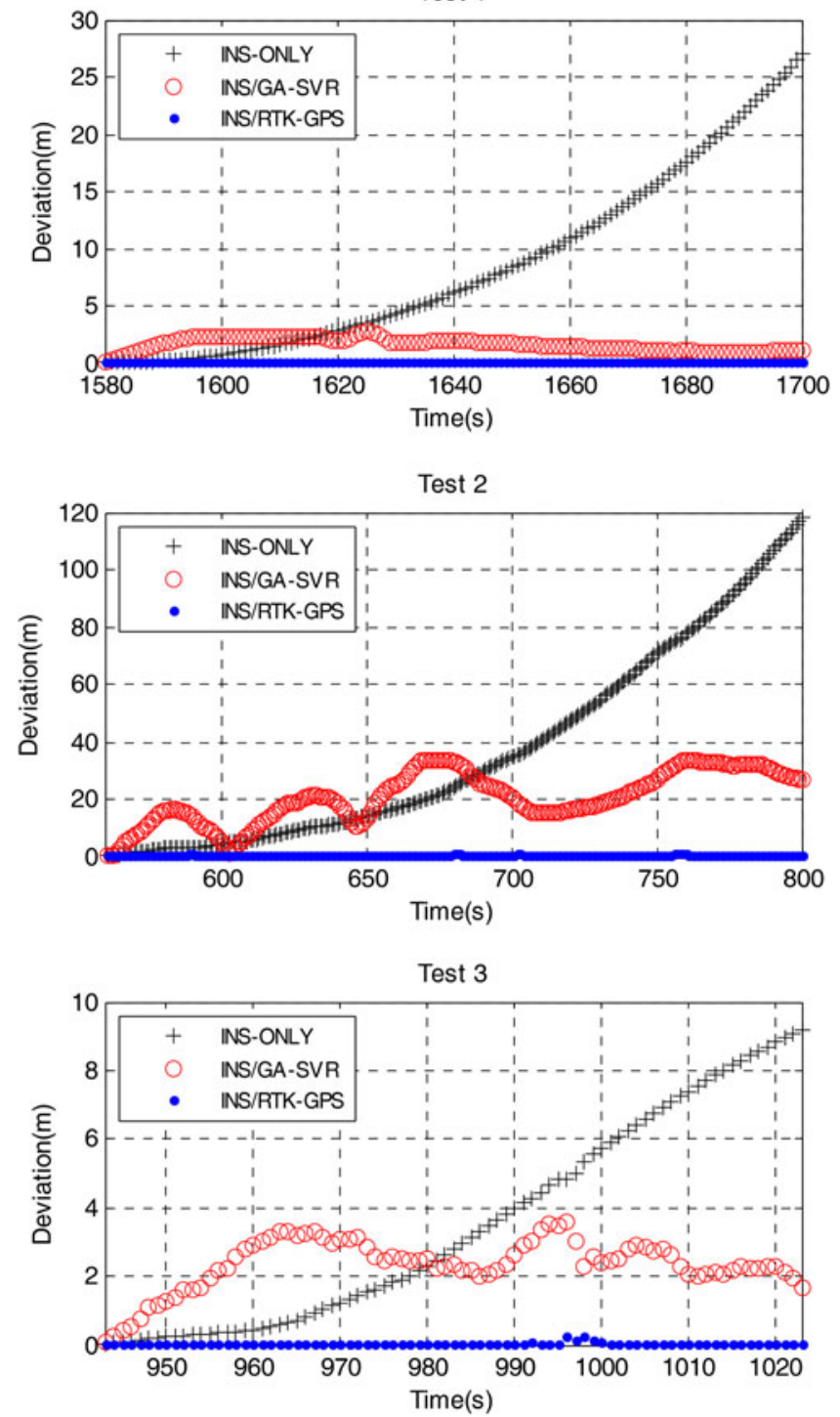

Figure 10. Position errors comparison in three dimensions. (a) Test 1 (Top). (b) Test 2 (Middle). (c) Test 3 (Bottom).

INS-only after that point as the result of the accuracy of the pseudo-GPS position. In Test 3, deviations in the INS-only drift to $9 \mathrm{~m}$ in 81 seconds, while the RMS of the deviation with INS/GA-SVR is $2.472 \mathrm{~m}$, with a maximum deviation of no more than $3.600 \mathrm{~m}$.

Figure 11 shows the velocity comparison for the INS/RTK-GPS and INS/GA-SVR algorithms in the north, east, and up directions. In Test 1, the RMS of the deviation using the INS/GA-SVR algorithm are $0.051 \mathrm{~m} / \mathrm{s}, 0.068 \mathrm{~m} / \mathrm{s}$, and $0.015 \mathrm{~m} / \mathrm{s}$, with the maximum no more than $0 \cdot 146 \mathrm{~m} / \mathrm{s}, 0 \cdot 187 \mathrm{~m} / \mathrm{s}$, and $0.041 \mathrm{~m} / \mathrm{s}$ in the north, east, and 

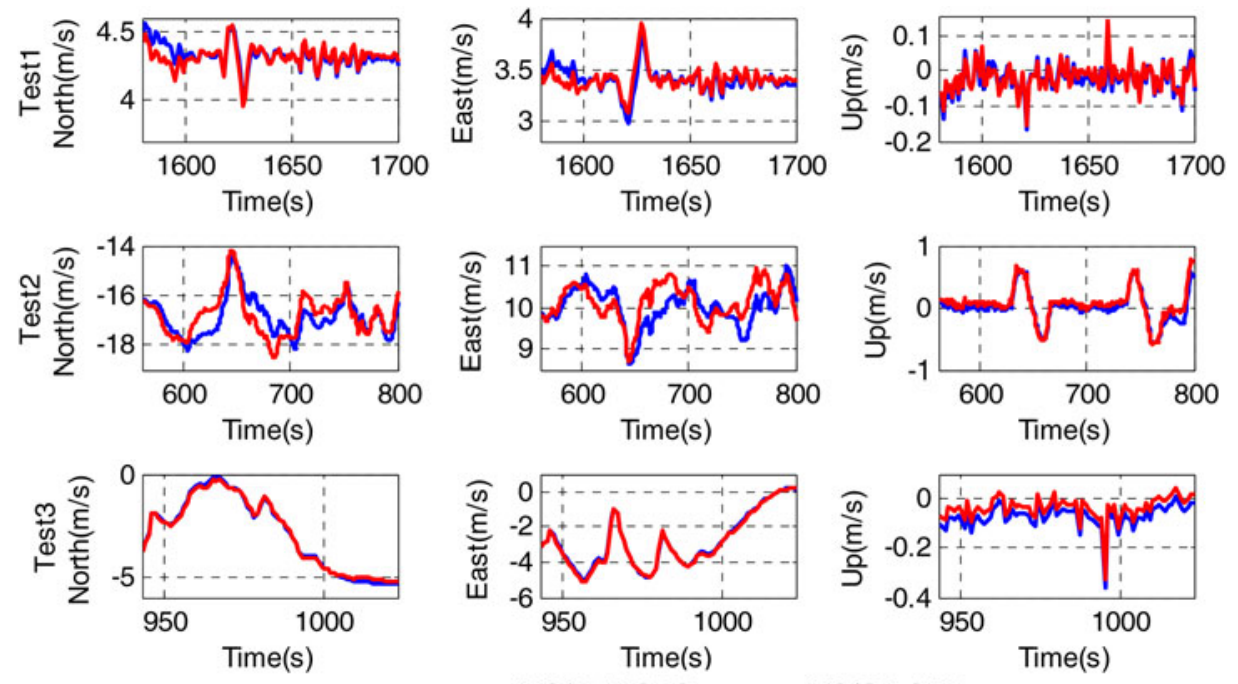

Figure 11. Velocity comparison. (a) Test 1 (Top). (b) Test 2 (Middle). (c) Test 3 (Bottom).

up directions, respectively. This is almost identical to the true velocity with INS/RTKGPS. In Test 2, the RMS of the deviation using the INS/GA-SVR algorithm are 0.560 $\mathrm{m} / \mathrm{s}, 0.406 \mathrm{~m} / \mathrm{s}$, and $0.075 \mathrm{~m} / \mathrm{s}$, with a maximum of no more than $1.003 \mathrm{~m} / \mathrm{s}, 0.782 \mathrm{~m} / \mathrm{s}$, and $0.226 \mathrm{~m} / \mathrm{s}$ in north, east, and up directions, respectively. In Test 3, the RMS of the deviation using the INS/GA-SVR algorithm are $0.104 \mathrm{~m} / \mathrm{s}, 0.077 \mathrm{~m} / \mathrm{s}$, and $0.033 \mathrm{~m} / \mathrm{s}$, with a maximum deviation of less than $0.239 \mathrm{~m} / \mathrm{s}, 0.187 \mathrm{~m} / \mathrm{s}$, and $0.040 \mathrm{~m} / \mathrm{s}$ in north, east, and up directions, respectively. This result indicates that the velocity in the up direction is close to that with INS/RTK-GPS, but there are small deviations between INS/RTK-GPS and INS/GA-SVR in the north and east directions.

Figure 12 shows an attitude comparison of the INS/RTK-GPS and INS/GA-SVR algorithms in the roll, pitch, and heading. In Test 1, the RMS of the deviation with the INS/GA-SVR algorithm are $0.109^{\circ}, 0.250^{\circ}$, and $0.222^{\circ}$, with the maximum no more than $0.161^{\circ}, 0 \cdot 342^{\circ}$, and $0.290^{\circ}$ in the roll, pitch, and head, respectively, which are almost identical to the attitude of INS/RTK-GPS. In Test 2, the RMS of the deviation with the INS/GA-SVR algorithm are $0.039^{\circ}, 0.135^{\circ}$, and $0.985^{\circ}$, with a maximum of no more than $0.088^{\circ}, 0.278^{\circ}$, and $1.509^{\circ}$ in the roll, pitch, and head, respectively. In Test 3 , the RMS of the deviation with the INS/GA-SVR algorithm are $0 \cdot 023^{\circ}, 0 \cdot 040^{\circ}$, and $0 \cdot 111^{\circ}$, with a maximum of no more than $0.058^{\circ}, 0.070^{\circ}$, and $0 \cdot 168^{\circ}$ in the roll, pitch, and head, respectively. These results indicate that the attitude in the roll and pitch is close to INS/RTK-GPS, but there are small differences between INS/RTK-GPS and INS/GA-SVR in the heading. The statistical results for the velocity and attitude are given in Table 3.

The results indicate that 1) Based on the GA-SVR algorithm, the accuracy of the pseudo-GPS position is high when the vehicle operates a low-speed-stable navigation platform along both a straight line and a curve, and low if the vehicle operates a high-speed-unstable navigation platform; 2) The velocity and attitude of navigation solutions are very close to the true value, and they benefit from the implementation 

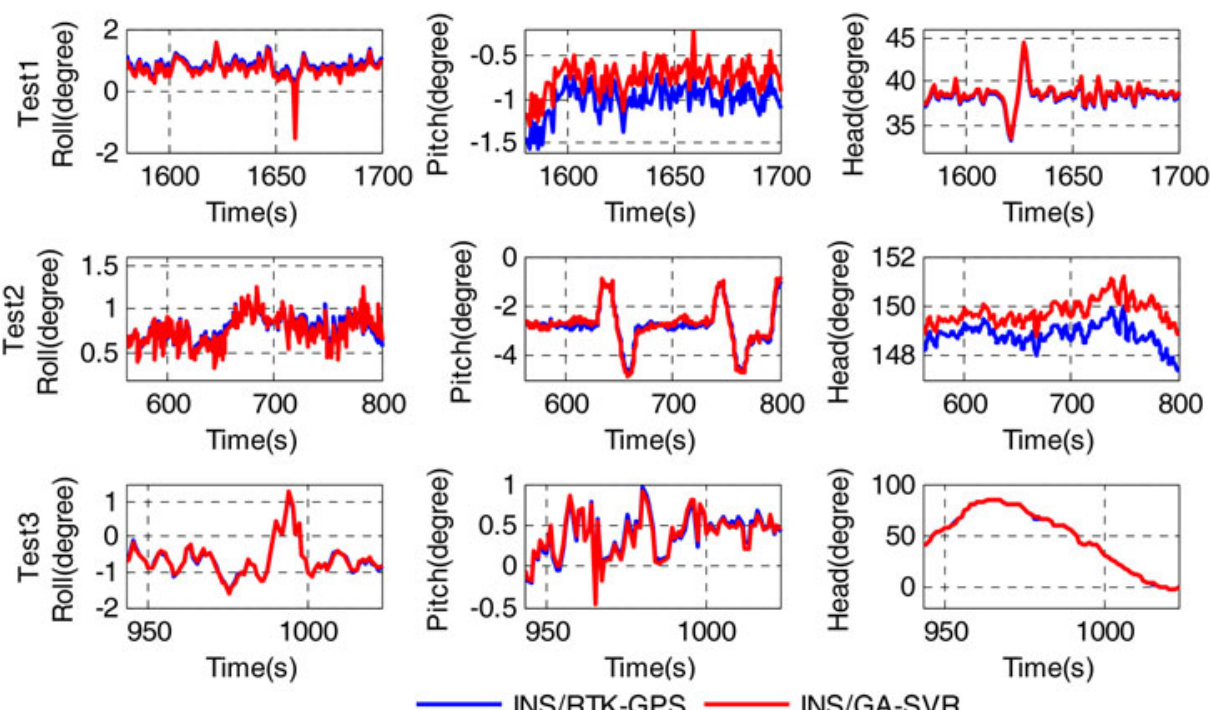

Figure 12. Attitude comparison. (a) Test 1 (Top). (b) Test 2 (Middle). (c) Test 3 (Bottom).

Table 3. Results of velocity and attitude comparison.

\begin{tabular}{|c|c|c|c|c|c|c|c|}
\hline \multirow[b]{2}{*}{ Test } & & \multicolumn{3}{|c|}{ Velocity deviation $(\mathrm{m} / \mathrm{s})$} & \multicolumn{3}{|c|}{ Attitude deviation (degree) } \\
\hline & & North & East & $\mathrm{Up}$ & Roll & Pitch & Head \\
\hline \multirow[t]{2}{*}{1} & Max & $0 \cdot 146$ & $0 \cdot 187$ & $0 \cdot 041$ & $0 \cdot 161$ & $0 \cdot 342$ & $0 \cdot 290$ \\
\hline & RMS & $0 \cdot 051$ & $0 \cdot 068$ & $0 \cdot 015$ & $0 \cdot 109$ & $0 \cdot 250$ & $0 \cdot 222$ \\
\hline \multirow[t]{2}{*}{2} & Max & $1 \cdot 003$ & $0 \cdot 782$ & $0 \cdot 226$ & $0 \cdot 088$ & $0 \cdot 278$ & $1 \cdot 509$ \\
\hline & RMS & $0 \cdot 560$ & $0 \cdot 406$ & $0 \cdot 075$ & $0 \cdot 039$ & $0 \cdot 135$ & 0.985 \\
\hline \multirow[t]{2}{*}{3} & $\operatorname{Max}$ & $0 \cdot 239$ & $0 \cdot 187$ & $0 \cdot 040$ & $0 \cdot 058$ & $0 \cdot 070$ & $0 \cdot 168$ \\
\hline & RMS & $0 \cdot 104$ & $0 \cdot 077$ & 0.033 & $0 \cdot 023$ & $0 \cdot 040$ & $0 \cdot 111$ \\
\hline
\end{tabular}

of improved adaptive filtering that only adjusts the position covariance matrix rather than other states; and 3) The accuracy of navigation solutions depends largely upon the accuracy of the pseudo-GPS position when the GPS is unavailable.

6. CONCLUSIONS. To overcome the shortcomings of GPS/INS integrated navigation during GPS outages, we have proposed a pseudo-position-aided INS navigation algorithm. Based on GA-SVR, the algorithm uses the predicted pseudo-position and improved adaptive filtering to calculate reliable navigation solutions. The proposed algorithm has been tested on low-speed-stable and high-speed-unstable navigation platforms where the vehicle travels along a straight line and around a curve. Results show that the proposed new approach can provide reliable and accurate navigation solutions when the GPS is unavailable. The calculation of the optimal parameters in an SVR training model will take more time, which will affect the real-time navigation of the integrated system. Thus the optimal parameters should be trained in advance according to the INS performance and the different motion states of the vehicle. With the 
further development of parallel computing technology and computer performance, this issue will be resolved in the future.

\section{ACKNOWLEDGMENTS}

The work is partially sponsored by the Program for New Century Excellent Talents in University (grant number: NCET-13-1019), partially sponsored by the Fundamental Research Funds for the Central Universities (grant number: 2013RC16); and partially sponsored by A Project Funded by the Priority Academic Program Development of Jiangsu Higher Education Institutions.

\section{REFERENCES}

Abdel-Hamid, W., Noureldin, A. and El-Sheimy, N. (2007). Adaptive fuzzy prediction of low-cost inertialbased positioning errors. Fuzzy Systems, IEEE Transactions on, 15, 519-529.

Alpaydin, E. (2004). Introduction to machine learning. MIT press.

Bar-Shalom, Y., Li, X.R. and Kirubarajan, T. (2001). Estimation with applications to tracking and navigation. John Wiley \& Sons.

Berk, R. (2008). Support Vector Machines. Springer, New York.

Chang, Q., Chen, Q. and Wang, X. (2005). Scaling Gaussian RBF kernel width to improve SVM classification. Proceedings of the Neural Networks and Brain. ICNN\&B'05. International Conference on,

Chiang, K.-W., Duong, T.T., Liao, J.-K., Lai, Y.-C., Chang, C.-C., Cai, J.-M. and Huang, S.-C. (2012). OnLine Smoothing for an Integrated Navigation System with Low-Cost MEMS Inertial Sensors. Sensors, 12, 17372-17389.

Chiang, K.W., Noureldin, A. and El-Sheimy, N. (2003). Multisensor integration using neuron computing for land-vehicle navigation. GPS solutions, 6, 209-218.

Cristianini, N. and Ricci, E. (2008). Support Vector Machines. Springer US.

Danezis, C. and Gikas, V. (2013). An Iterative LiDAR DEM-Aided Algorithm for GNSS Positioning in Obstructed/Rapidly Undulating Environments. Advances in Space Research, 52, 865-878.

Ding, W., Wang, J., Rizos, C. and Kinlyside, D. (2007). Improving adaptive Kalman estimation in GPS/INS integration. Journal of Navigation, 60, 517-529.

El-Sheimy, N., Chiang, K. and Noureldin, A. (2008). Developing a low cost MEMS IMU/GPS integration scheme using constructive neural networks. IEEE Trans. Aerosp. Electron. Syst, 44, 582-594.

Falco, G., Einicke, G., Malos, J. and Dovis, F. (2012). Performance Analysis of Constrained Loosely Coupled GPS/INS Integration Solutions. Sensors, 12, 15983-16007.

Farrell, J. (2008). Aided navigation: GPS with high rate sensors. McGraw-Hill, New York.

Faruqi, F.A. and Turner, K.J. (2000). Extended Kalman filter synthesis for integrated global positioning/ inertial navigation systems. Applied mathematics and computation, 115, 213-227.

Frangos, K., Kealy, A., Gikas, V. and Hasnur, A. (2010). Dynamic modeling for land mobile navigation using low-cost inertial sensors and least squares support vector machine learning. Proceedings of the Proceedings of the 23rd International Technical Meeting of The Satellite Division of the Institute of Navigation (ION GNSS 2010).

Geng, Y., Deurloo, R. and Bastos, L. (2011). Hybrid derivative-free extended Kalman filter for unknown lever arm estimation in tightly coupled DGPS/INS integration. GPS solutions, 15, 181-191.

Gikas, V., Cross, P. and Akuamoa, A. (1995). A rigorous and integrated approach to hydrophone and source positioning during multi-streamer offshore seismic exploration. The Hydrographic Journal, 77, 11-24.

Goldberg, D.E. and Holland, J.H. (1988). Genetic algorithms and machine learning. Machine learning, 3, 95-99.

Gunn, S.R. (1998). Support vector machines for classification and regression. ISIS technical report, 14, $41-46$

Hewitson, S. and Wang, J. (2007). GNSS receiver autonomous integrity monitoring with a dynamic model. Journal of Navigation, 60, 247-263.

Hewitson, S. and Wang, J. (2010). Extended Receiver Autonomous Integrity Monitoring (e RAIM) for GNSS/INS Integration. Journal of Surveying Engineering, 136, 13-22. 
Huang, Y.W. and Chiang, K.W. (2008). An intelligent and autonomous MEMS IMU/GPS integration scheme for low cost land navigation applications. GPS solutions, 12, 135-146.

Joachims, T. (2002). Support Vector Machines. Springer, US.

Kecman, V. (2005). Support Vector Machines - An Introduction. Springer, Berlin Heidelberg.

Lu, P., Zhao, L. and Chen, Z. (2007). Improved Sage-Husa Adaptive Filtering and Its Application [J]. Journal of System Simulation, 15, 3503-3505.

Noureldin, A., Osman, A. and El-Sheimy, N. (2004). A neuro-wavelet method for multi-sensor system integration for vehicular navigation. Measurement science and technology, 15, 404-412.

Parnian, N. and Golnaraghi, F. (2010). Integration of a multi-camera vision system and strapdown inertial navigation system (SDINS) with a modified kalman filter. Sensors, 10, 5378-5394.

Sage, A. P. and Husa, G. W. Adaptive filtering with unknown prior statistics. (1969). Proceedings of the Proceedings of the Joint Automatic Control Conference, Tokyo, Japan, 760-769.

Semeniuk, L. and Noureldin, A. (2006). Bridging GPS outages using neural network estimates of INS position and velocity errors. Measurement science and technology, 17, 2783-2789.

Tang, Y., Wu, Y., Wu, M., Wu, W., Hu, X. and Shen, L. (2009). INS/GPS integration: global observability analysis. Vehicular Technology, IEEE Transactions on, 58, 1129-1142.

Van Diggelen, F. S. T. (2009). A-GPS: Assisted GPS, GNSS, and SBAS. Artech House.

Vapnik, V. (2000). The nature of statistical learning theory. Springer.

Wang, J. J., Wang, J., Sinclair, D. and Watts, L. (2006). A neural network and Kalman filter hybrid approach for GPS/INS integration. Proceedings of the 12th IAIN Congress \& 2006 Int. Symp. on GPS/ GNSS, Jeju, Korea,

Williams, G. (2011). Support Vector Machines. Springer, New York.

Wolfe, P. (1961). A duality theorem for nonlinear programming. Quarterly of applied mathematics, 19, 239-244.

Xu, Z., Li, Y., Rizos, C. and Xu, X. (2010). Novel hybrid of LS-SVM and kalman filter for GPS/INS integration. Journal of Navigation, 63, 289-299.

Yuanxi, Y. (1994). Robust estimation for dependent observations. Manuscripta geodaetica, 19, 10-17. 